\title{
Analysis of Composition and Morphology of Cuticular Wax of Wheat at Different Development Stages
}

\author{
Jiahuan Wang*, Jialin Fan, Bao'an Yang, Xiaojie Chen, Fuyan Zhang, Zhongjie Cheng and Jianwei Zhang* \\ Isotope Institute Co., Ltd, Henan Academy of Sciences/Henan Key Laboratory of Nuclear Agricultural Sciences, Zhengzhou, \\ Henan 450015, China \\ *For correspondence: m18292459524@163.com; zjw10308@163.com; wangjiahuan3@126.com \\ Received 16 September 2020; Accepted 28 December 2020; Published 25 March 2021
}

\begin{abstract}
In order to investigate the morphology and composition of cuticular waxes on the spikes and leaves at different plant growth stages, the glossy variety (Changwu9945-10) and glaucous variety (L955195) of wheat (Triticum aestivum) were selected. Scanning electron microscopy (SEM) revealed that the morphology of cuticular wax crystals changed constantly throughout plant growth, and the wax crystals of spike (glume) and leaf surfaces were tubules and platelets. Tubule wax crystals could formed on the glumes within a few days, but took longer time to form on the leaf surface. Gas Chromatography-Mass Spectrometry (GC-MS) analysis displayed five different compound classes on the extracts of the spikes and leaves, including alkane homologues (C25-C33), alcohol homologues (C22-C32), aldehyde homologue (C24-C30) and diketones (C31). Furthermore, the content of five compound classes changed at the different development stages in the cultivars. The total wax content at each development stage of L955195 was higher than that of Changwu9945-10. Interestingly, OH- $\beta$-diketones identified only in the glaucous variety (L955195). Diketone was the major compound class that caused the changes during the spike growth period, while alcohols were the major compound classes that caused the changes during the leaf growth period. At the same time, there were also dramatic changes in the chain length distribution of these compositions. Therefore, based on the above results, this study provided comprehensive information for wheat cuticular waxes and advanced the knowledge for development of wheat waxes in the future. (C) 2021 Friends Science Publishers
\end{abstract}

Keywords: Cuticular waxes; Wheat; GC-MS; Morphology; Diketones; Spike; Leaf

\section{Introduction}

The terrestrial plants surfaces are covered by the cuticle, and the cuticle is made up of cuticular waxes and a cutin polymer matrix (Jeffree 2006). Cuticular waxes are of interest to biologist as they have multiple roles in plants, such as insect destruction, protection of plants against ultraviolet (UV) radiation, restricts non-stomatal water loss and pests attack (Eigenbrode and Espelie 1995; Solovchenko and Merzlyak 2003; Domínguez et al. 2011).

The epidermal wax is complex mixtures, and is composed of very-long-chain fatty acids (VLCFAs, Chain length $>$ C20), including, alkanes, alcohols, ketones, aldehydes, esters and fatty acids (Jetter et al. 2006; Wang et al. 2020). Other components are also found in wax mixtures, such as p-hydroxycinnamic acids, monoacylglycerols (Li et al. 2007), flavonoids (Samuels et al. 2008), alkylresorcinols (Adamski et al. 2013), benzyl, phenethyl esters (Rapley et al. 2004) and triterpenoids (Javelle et al. 2011; Belge et al. 2014; Chai et al. 2018). The cuticular wax compositions of plants are different in different species. For example, in wheat, diketones, ester, alkanes, aldehydes and alcohols are the major compositions of the glume cuticular waxes (Wang et al. 2015a). However, on tomato leaves, the cuticular waxes are mainly composed of n-alkanes, branched alkanes, primary alcohols and triterpenoids (Wang et al. 2015b).

The leaves, stems, and fruits of land plants are coated by cuticles. In most cases, cuticular wax is usually present on the surface of plants in the form of microcrystals. In the past 60 years, scanning electron microscopy (SEM) has been widely used in observing the morphology of wax crystals (Koch and Ensikat 2008). Twenty-three wax crystal types have been identified from 13,000 species. In the past few years, several studies have examined the wax crystal of wheat leaf surfaces. Koch et al. (2006) observed platelets wax crystals on the two-month-old wheat leaves (Koch et al. 2006). Tubule wax crystals have been observed on the wheat glume during grain filling period (Wang et al. 2015a). Although the composition of epidermis wax and wax crystal have been studied in wheat (Bianchi and Figini 1986; Adamski et al. 2013; Zhang et al. 2013; Wang et al. 2015a; Koch et al. 2006), it remains unclear as to at which the developmental changes in the cuticular wax composition and crystals occur in various wheat. 
In this paper, GC-MS analyses were carried out to investigate the differences in the cuticular wax patterns of spikes and leaves in different development periods of two wheat varieties Changwu9945-10 (glossy variety) and L955195 (glaucous variety). At the same time, SEM was applied to investigate the morphology of cuticular wax at different development stages. This study could provide the wax development patterns of wheat and contribute to the further study of developing wheat with desired contents of cuticular waxes.

Although there were some studies on wheat epidermis waxes, many of them studies paid close attention to either whole plants or only leaves of various wheat cultivars without distinguishing the development changes of the wheat plant (Bianchi et al. 1980), only the latter research quantified wax loads per surface area (Racovita et al. 2007; Wang et al. 2015a; Li et al. 2019). This is the first detailed and comprehensive (compositional and morphological) comparisons of cuticular waxes from different development stages of the two wheat cultivars.

\section{Materials and Methods}

\section{Plant materials and reagents}

Plant materials: Wheat materials were provided by College of Agronomy, Northwest A\&F University, Yangling, Shaanxi, China. They were grown in the research field of Xinzheng, Henan province of China (3439'N 113⒌'E) from October 2019 to May 2020. Ten seeds of each variety were hand-planted in a $1 \mathrm{~m}$ row at $10 \mathrm{~cm}$ spacing. The average precipitation, minimum and maximum temperatures from October $8^{\text {th }} 2019$ to May $10^{\text {th }} 2020$ in this region were $257.8 \mathrm{~mm},-7^{\circ} \mathrm{C}$ and $39^{\circ} \mathrm{C}$. Changwu9945-10 and L955195 were selected as experimental materials because of their different waxy phenotypes. At the same time, they had winter growth habit and similar growth periods. The natural precipitation could meet the conditions of wheat growth. Plants were fertilized every three months. Spike samples were excised randomly from three individual plants at 1,2 , 4, 6, 8 and 14 days after heading (DAH) using clean razor blades in April 2020. Leaf samples were also excised randomly from three individual plants at 50, 100, 200 and 230 days of wheat ages during the 2019-2020 wheatgrowing seasons. Exact weights of spikes were taken by weighing after extraction (dry weight). Exact areas of leaf were determined by photographing them, and then the area was calculated by the ImageJ software.

\section{Reagents and instruments}

Chloroform (Yingfeng, China) was used to extract cuticular waxes from wheat samples. N,O-bis (trimethylsilyl)trifluoroacetamide (BSTFA) (Sigma, USA) and pyridine (Sigma, USA) were used for derivatization reactions. Dry nitrogen blowing apparatus (LC-DCY-12G, Lichen, China) was used for rapid evaporation of derivatization reaction products. SEM (Hitachi S4800, Tokyo, Japan) was used for investigated wax crystals. GC-MS (QP2010, Shimadzu, Japan) and GC-FID (7890B, Agilent, USA) were applied to identify and determine the composition of cuticular waxes of the wheat samples, respectively.

\section{Scanning electron microscopy (SEM)}

As the spikes of wheat were covered by glumes, we investigated cuticular wax crystals of glume surfaces by SEM. Glumes were sampled at 1, 2, 4, 6, 8 and $14 \mathrm{DAH}$, and leaves were sampled at the 50, 100, 200 and 230 days. All the samples were dried for three days at $60^{\circ} \mathrm{C}$ in a desiccator. Three $\mathrm{mm}^{2}$ dried sample was mounted onto SEM tubes, then coated with gold particles from a sputter coater (Hitachi E-1045, Japan) (Wang et al. 2017). Coated samples were observed using SEM $(10 \mathrm{kV}$ accelerating voltage; $8.5 \mathrm{~mm}$ working distance), and each coated sample was detected at $30,000 \times$ and 10,000× objective, respectively.

\section{Extraction of cuticular wax from the spikes and leaves}

Each sample was immersed quickly in a glass beaker containing $40 \mathrm{~mL}$ chloroform $\left(\mathrm{CHCl}_{3}\right), 10 \mu \mathrm{L}$ n-tetracosane $\mathrm{C} 24$ (concentration: $1 \mu \mathrm{g} / \mu \mathrm{L}$ ) was add into the mixture as an internal standard, and shaken twice for $30 \mathrm{~s}$ at $25^{\circ} \mathrm{C}$. After that, filtered the wax sample through a paper filter, transferred to a GC autosampler sample bottle, and dried under nitrogen flow.

\section{Derivatization reactions}

To transform hydroxyl (OH-) containing compounds into their corresponding trimethylsilyl derivatives (Schulz et al. 2000), each wax sample was treated with $50 \mu \mathrm{L}$ BSTFA and $50 \mathrm{~mL}$ pyridine at $70^{\circ} \mathrm{C}$, held for $1 \mathrm{~h}$. Samples of $\beta$ diketones and alcohols isolated from wheat leaves were derivatized as decribed previously (Adamski et al. 2013). Later on, the mixture sample was quickly dried in a dry nitrogen blowing apparatus with nitrogen gas flow, then added $700 \mu \mathrm{L} \mathrm{CHCl}_{3}$ for the $\mathrm{GC}$ analysis.

\section{Chemical analysis of cuticular waxes}

After derivatization, the wax samples were analyzed by GCMS and GC-FID (Falkland Islands Dependencies). GC-MS was used for qualitative analysis. The GC equipped with a HP-1 column (film thickness $0.25 \mu \mathrm{m}$, diameter $0.32 \mathrm{~mm}$, $30 \mathrm{~m}$ long; Agilent, USA) attached to an MS was used to analyze wax compositions of each samples, a rate of 2 $\mathrm{mL} / \mathrm{min}$ helium (He) was used as carrier gas. The temperature program of GC-MS was as follows: set at $50^{\circ} \mathrm{C}$ for $2 \mathrm{~min}$, increased to $220^{\circ} \mathrm{C}$ at a rate of $20^{\circ} \mathrm{C} / \mathrm{min}$, held for $2 \mathrm{~min}$, increased to $310^{\circ} \mathrm{C}$ at a rate of $1.6^{\circ} \mathrm{C} / \mathrm{min}$, held at $310^{\circ} \mathrm{C}$ for $18 \mathrm{~min}$. The GC-FID was used for composition quantitative analysis under the above GC conditions but with nitrogen $\left(\mathrm{N}_{2}\right)$ carrier gas. 


\section{Statistical analysis}

The load of each wheat wax component was calculated based on peak area of each compound and the peak area of C24. Every sample was determined in triplicates, and the coefficient of variance (CV) of the three samples was less than $10 \%$. Sigma plot 14.0 software was carried out to draw pictures in this paper.

\section{Results}

Morphological changes in wax crystal on the glumes of wheat

The glossy (Changwu9945-10) and glaucous (L955195) varieties of wheat were used in this paper. They were in different cuticular wax phenotype (Fig. 1). Because the spikes of wheat were covered by glumes, which we observed the glume surfaces by SEM. Two types of crystals were identified on the glume surfaces: platelets and tubules. Interestingly, the platelet crystals were presented only on the Changwu9945-10 glume surfaces, but tubule crystals were formed both on the glume surfaces of both the varieties (Fig. 2). For Changwu9945-10, on 1 and 2 DAH, only a few of platelet crystals were discovered on the glumes surfaces, and the glumes were covered by a relatively smooth film (Fig. 2). On 4 DAH, tubule crystals and platelet crystals were discovered for the first time, and this trend continued until 8 DAH (Fig. 2); On 14 DAH, platelet crystals completely disappeared and tubule crystals tended to be densest. Strikingly, on the glume surfaces of L955195, there were already a small amount of tubule crystals identified on the 1 DAH (Fig. 2). On 2 DAH, L955195 glumes were covered with more denser tubule crystals (Fig. 2) and this trend continued from 2 to 14 DAH. L955195 displayed a denser array of tubule crystals than that of Changwu9945-10 during the spike development period. This suggested different modes of the development of wax crystals on glumes in glossy and glaucous varieties.

\section{Morphological changes in wax crystal on the leaf surfaces of wheat}

Compared with wax crystals on the glumes, wax crystals on the leaf surfaces displayed different development patterns (Fig. 3). From 50 to $100 \mathrm{~d}$, both the leaf surfaces of Changwu9945-10 and L955195 were covered with platelet crystals (Fig. 3). Interestingly, on the $200 \mathrm{~d}$, tubule crystals were noted on the leaf surfaces of L955195, while all crystals disappeared on the leaf surfaces of Changwu994510. On the $230 \mathrm{~d}$, the wax crystal remained the same as on 200 d (Fig. 3). During the leaf development period, L955195 displayed a very drastic change in wax crystal morphology, which changed from platelet to tubule. However, Changwu9945-10 showed a different change in crystal morphology, platelet crystals only maintained for 100 days, then disappeared as time went on (Fig. 3).

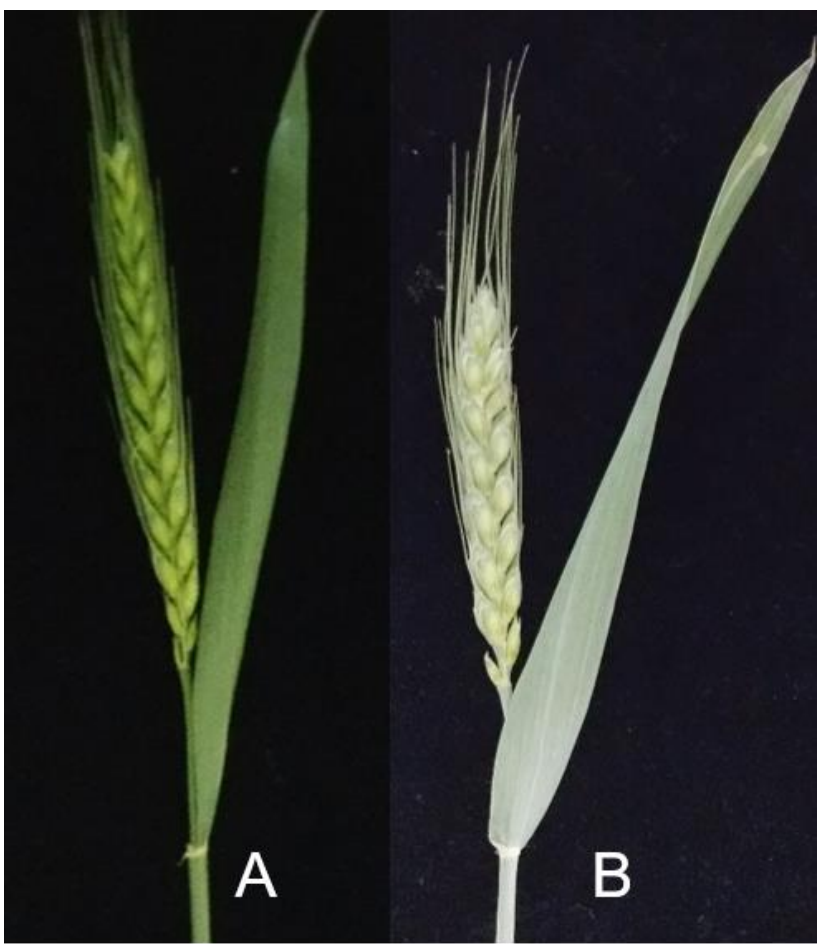

Fig. 1: Cuticular wax phenotype on the spike of the two wheat varieties. Glossy variety of Changwu9945-10 (A), and glaucous variety of L955195 (B)

\section{Changes in the wax composition on the spikes during the} spike development

GC chromatogram displayed that the spikes of Changwu9945-10 and L955195 consisted of 15 compounds, including alkanes, alcohols, aldehydes and diketones (Fig. 4). The total content of wax on the spike of two wheat varieties increased from 1 to $8 \mathrm{DAH}$, and then decreased from 8 to $14 \mathrm{DAH}$ (Fig. 5A). The total content wax of Changwu9945-10 spikes was 160.17, 546.44, 834.14, $1089.24,1694.62$ and $1114.56 \mu \mathrm{g} / \mathrm{g}$ at $1,2,4,6,8$ and 14 DAH, respectively (Fig. 5A). In the spike of L955195, diketones were in the leading place (Fig. 5B), while alkanes were the major constituent in the spike wax of Changwu9945-10 (Fig. 5A). The total content of alkanes and diketones on the spikes also increased continuously from 1 to $8 \mathrm{DAH}$, and then decreased (Fig. 5). During the spike development, the content of aldehydes and alcohols showed slight fluctuations (Fig. 5).

At the same time, the homologs of each compound class also tend to be with the same regular pattern (Fig. 6). Diketones were identified as $\beta$-diketone and $\mathrm{OH}-\beta$-diketone. Total content of $\mathrm{OH}-\beta$-diketones on glaucous variety L955195 spikes was 162.0, 1101.7, 1408.8, 1844.6, 2477.1 and $1788.0 \mu \mathrm{g} / \mathrm{g}$ at $1,2,4,6,8$ and $14 \mathrm{DAH}$, respectively. Interestingly, no $\mathrm{OH}-\beta$-diketone was detected in Changwu9945-10 (Fig. 6A), while very high content of $\mathrm{OH}-$ $\beta$-diketone was detected in L955195 (Fig. 6B). The carbon 


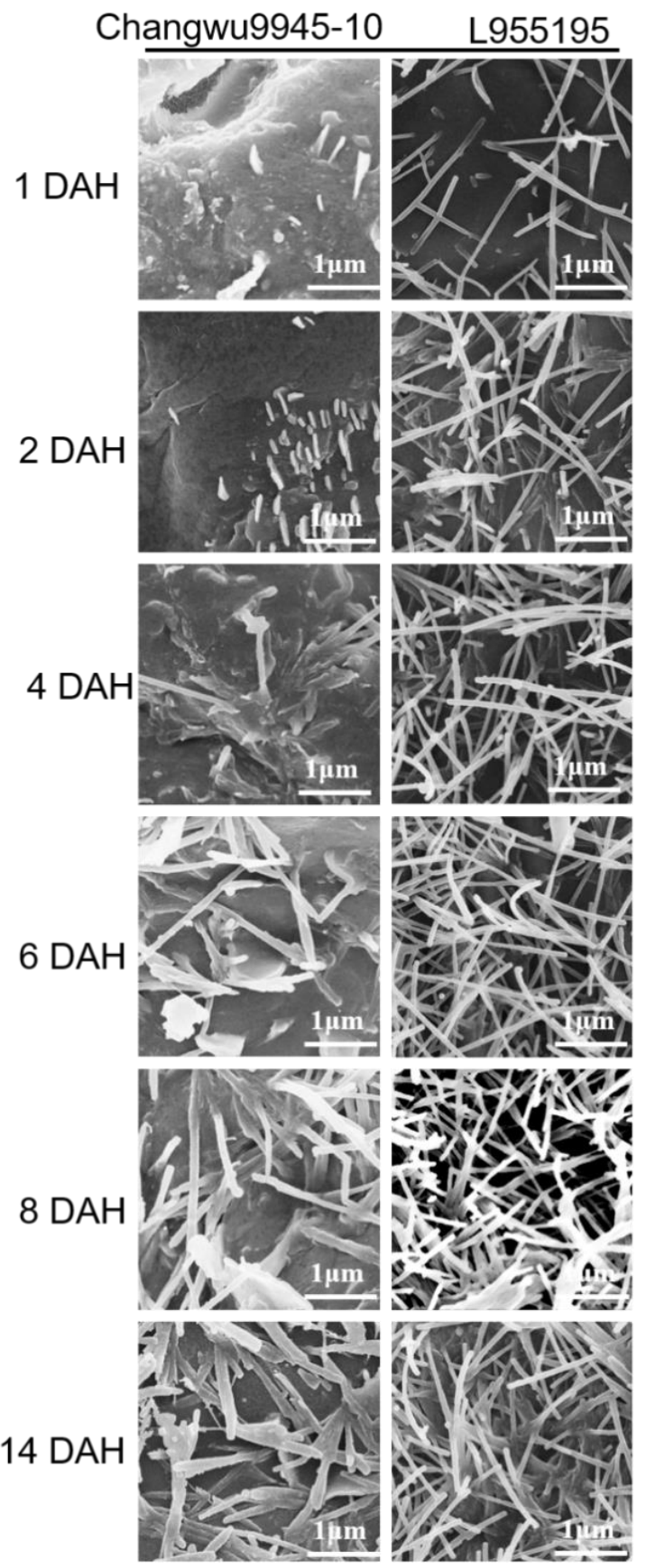

Fig. 2: Developmental changes of epicuticular wax crystals on the glume surfaces of Changwu9945-10 and L955195. The six stages of plant development are indicated on the left. The micrographs are at a resolution of $10000 \times$, and the bars indicate $1 \mu \mathrm{m}$

chain length of alkanes ranged from $\mathrm{C} 25$ to $\mathrm{C} 33$, and its distribution was relatively wide and odd. C29 and C31 were the dominant alkanes during the spike development period. A series of alcohols (C22 to C32) were also identified (C32 not detected in L955195), with C26 or C28 being the most prominent. It is worth noting that L955195 yielded high amounts of C26 and C28, while Changwu9945-10 yielded

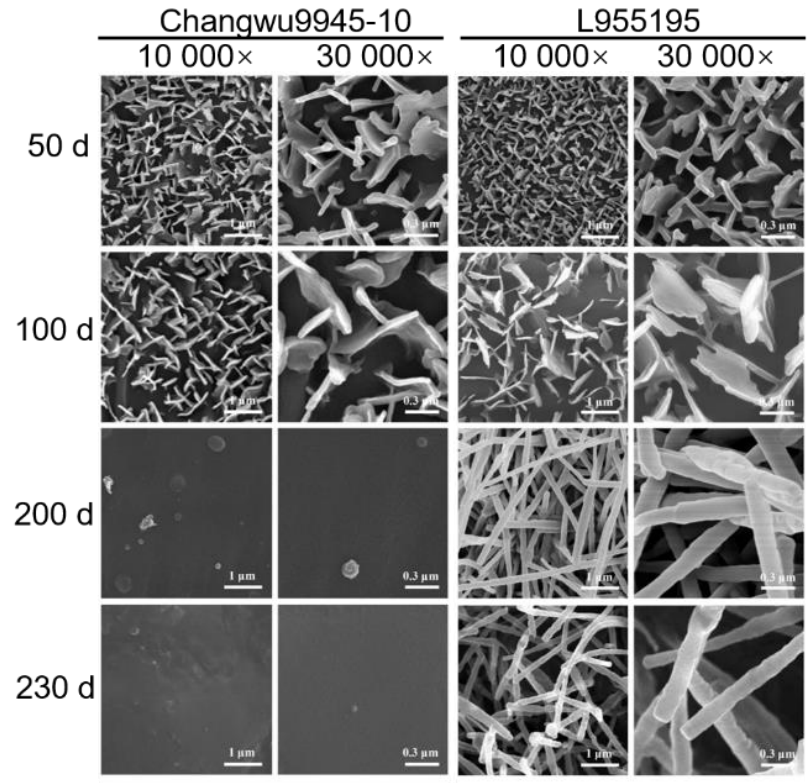

Fig. 3: Developmental changes of epicuticular wax crystals on the leaf surfaces of Changwu9945-10 and L955195. The four stages of plant development are indicated on the left. The magnification of each column are labeled on the top. The micrographs are at a resolution of $10000 \times$ and $30000 \times$, and the bars indicate $1 \mu \mathrm{m}$ and $0.3 \mu \mathrm{m}$, respectively

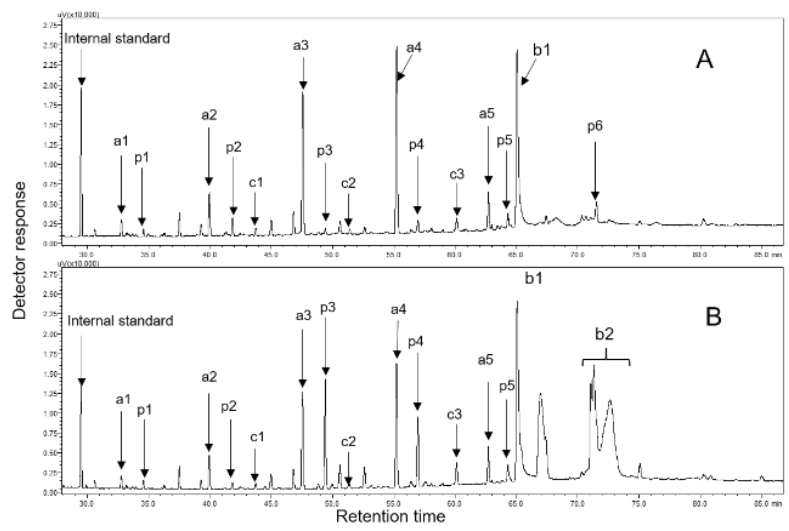

Fig. 4: The GC chromatogram of the spikes of Changwu9945-10 (A) and L955195 (B). Internal standard (C24); a1-a5, alkanes; a1, pentacosane (C25); a2, heptacosane (C27); a3, nonacosane (C29); $\mathrm{a} 4$, hentriacontane (C31); a5, tritriacontane (C33); p1-p6, alcohols; p1,docosanol (C22); p2, tetracosanol (C24); p3, hexacosanol (C26); p4, octacosanol (C28); p5, triacontanol (C30); p6, dotriacontanol (C32); c1-c3, aldehydes; c1, hexacosanal (C26); c2, octacosanal (C28); c3, triacontanal (C30); b1-b2, Diketones, b1, $\beta$-diketone (C31); b2, OH- $\beta$-diketone (C31)

minor amounts (Fig. 6). In addition, a series of aldehydes (C26 to C30) were also identified, although in lower amounts. Results showed that the homologs of each compound class increased continuously from 1 to $8 \mathrm{DAH}$, then decreased from 8 to 14 DAH (Fig. 6). 

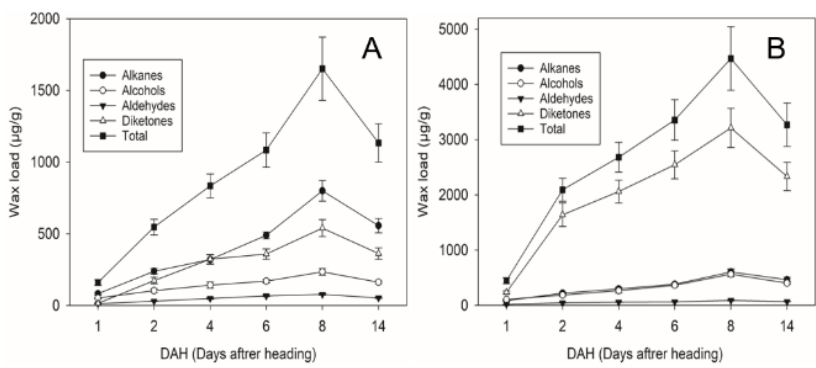

Fig. 5: Development changes in the spike surface wax component and the total wax load. (A) Changwu9945-10, (B) L955195. Spike surface wax was measured at six stages $(1,2,4,6,8$ and 14 DAH). Wax coverage is expressed as $\mu \mathrm{g} / \mathrm{g}$ of wheat spikes dry weight. Each datum point represents a pooled sampled of at least three spikes. Each value represents the mean of three replicates. Error bars $=$ SD. The amounts of diketones is the sum of $\beta$ - and $\mathrm{OH}-\beta$-diketones

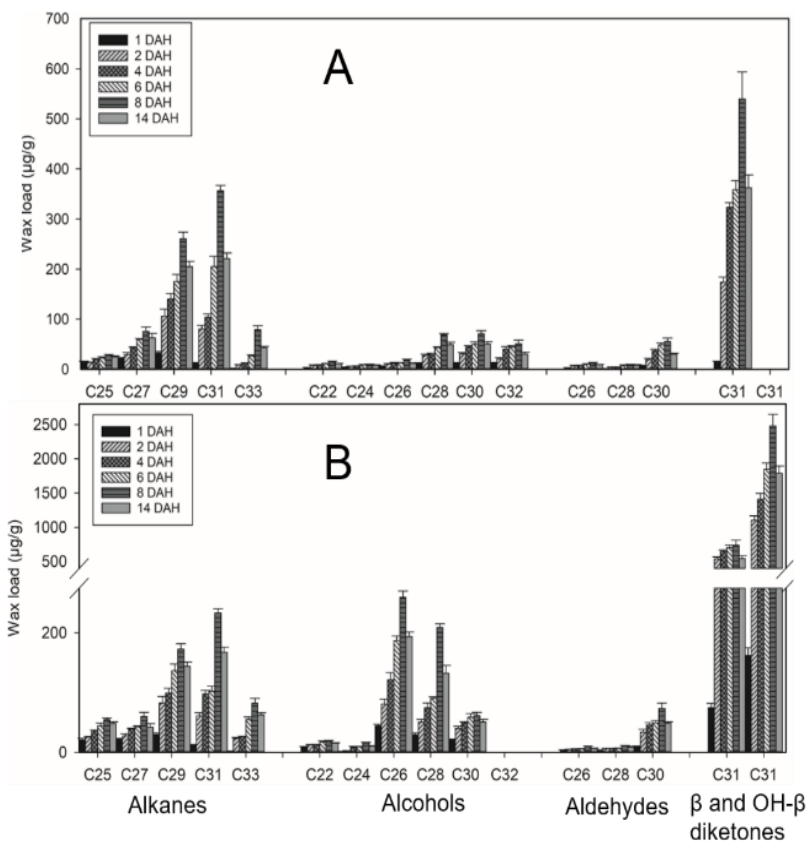

Fig. 6: Chain length distribution in the individual wax constituent on the spikes. (A) Changwu9945-10, (B) L955195. Each wax constituent is designated by carbon chain length and is labeled by chemical class along the $\mathrm{x}$-axis. Each value represents the mean of three replicates. Error bars $=\mathrm{SD}$

\section{Changes in the wax composition on the leaves during the leaf development}

There were five compounds in the leaf wax of the two wheat varieties, including alkanes, alcohols, aldehydes, and diketones, they were the same as wax composition of spikes (Fig. 7). The cuticular waxes on the leaves showed different developmental regular patterns between the two wheat varieties. For instance, the total content of wax on the L955195 leaves increased continuously during the wheat
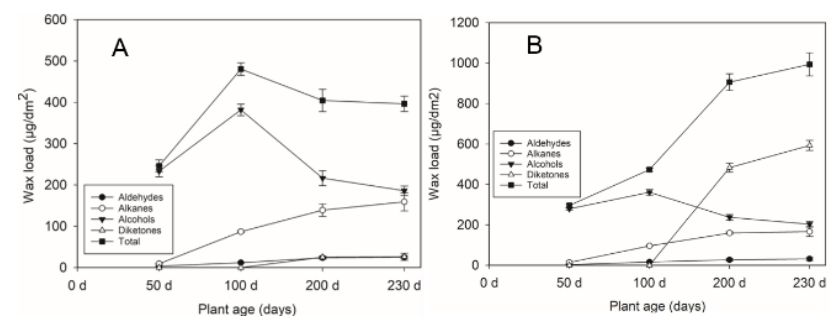

Fig. 7: Developmental changes in wax component and the total load on the leaf surfaces. (A) Changwu9945-10, (B) L955195. Four representative developmental stages (50, 100, 200 and 230 days) were investigated for wax coverage. The absolute amounts of cuticular waxes are expressed as $\mu \mathrm{g} / \mathrm{dm}^{2}$ of leaf blade surface area. Each datum point represents a pooled sampled of at least three wheat leaves. Each value represents the mean of three replicates. Error bars $=\mathrm{SD}$
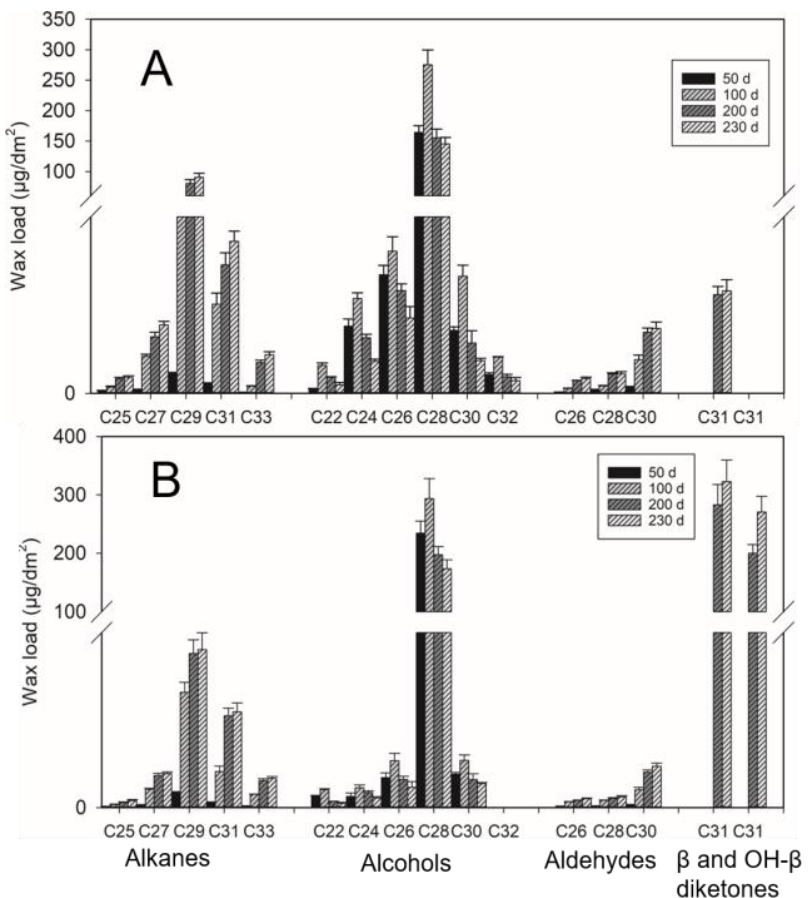

Fig. 8: Chain length distribution in the individual wax constituent on the leaf surfaces. (A) Changwu9945-10, (B) L955195. Each wax constituent is designated by carbon chain length and is labeled by chemical class along the $\mathrm{x}$-axis. Each value represents the mean of three replicates. Error bars $=$ SD

growth (Fig. 7B). However, on the Changwu9945-10 leaves, the total content wax increased from 50 to $100 \mathrm{~d}$, and then decreased from 200 to 230 d (Fig. 7A). From 50 to 100 d, alcohols were the major compound of the leaves cuticular waxes both in the Changwu9945-10 and L955195, the content of alcohols increased rapidly (Fig. 7). On $200 \mathrm{~d}$, the amount of alcohols decreased suddenly, this trend continued until 230 d. Contrarily, from 200 to 230 d, the content of alkanes, aldehydes and diketones increased steadily and diketones became the dominant compound of the leaves 
cuticular waxes, instead of alcohols (Fig. 7). All the above results revealed that diketones and alcohols were the major compound that contributed to the change of the total wax content during the plant growth period.

On the other hand, the homologs of each compound class also changed during the leaf development period. A series of alcohols (C22 to C32) were also identified, with octacosanol (C28) being the dominant homolog of alcohols. The content of octacosanol (C28) on the Changwu9945-10 leaves was $164.17,275.17,155.17$ and $145.17 \mu \mathrm{g} / \mathrm{dm}^{2}$ at 50 , 100,200 and 230d, respectively. Interestingly, dotriacontanol (C32) was not detected in L955195 (Fig. 8B), while no OH- $\beta$-diketone was detected in Changwu9945-10 (Fig. 8A). In addition, two series of alkanes (C25 to C33) and aldehydes (C26 to $\mathrm{C} 30$ ) were also identified in the leaf wax, with $\mathrm{C} 29$ and $\mathrm{C} 31$ being the dominant chain length of alkanes. The above results showed that the homologs of some compound classes (alkane homologues, aldehyde homologues and diketones) increased continuously from 50 to $230 \mathrm{~d}$ (Fig. 8), but alcohol homologues increased from 50 to $100 \mathrm{~d}$, then decreased from 100 to $230 \mathrm{~d}$ (Fig. 8).

\section{Discussion}

Epidermis wax layer covers the surface of the leaves, stems and spikes, giving the plant surface a glaucous or glossy appearance (Jenks and Ashworth 1999; Koch and Ensikat 2008). Previous researches indicated that tubule crystals are dominated by $\beta$-diketone, and the tubules mainly contribute to the glaucous phenotype in wheat (Bianchi and Figini 1986; Adamski et al. 2013; Zhang et al. 2013). Our results showed that Changwu9945-10 (glaucous variety) and L955195 (glossy variety) were covered with wax tubules on glume at 14 DAH (Fig. 2). However, the anomalistic wax film was present on the leaf surface of L955195 on $230 \mathrm{~d}$ (Fig. 3). The presence of diketones in all wax samples indicated that $\beta$-diketones could be identified both in the glaucous and glossy cultivars, while $\mathrm{OH}-\beta$-diketones could be only identified in the glaucous cultivar. These results provided an indication that the threshold value of $\beta$-diketone contents must be reached before tubule wax crystals present on the wheat surfaces. It also revealed that the content of diketone facilitated the morphological changes of wax crystal during the wheat development. For instance, when the $\beta$ diketones content reached to $282.78 \mu \mathrm{g} / \mathrm{dm}^{2}$, tubule wax crystals appeared on the leaf surface of Changwu9945-10 (Fig. 3). Our results also demonstrated the pattern of morphological changes of wax crystals, these tubule crystals on the leaf surface could be formed very slowly, while tubule and platelet crystals on glume were formed rapidly within a few days (Fig. 2-3).

The total content of epicuticular wax on the spikes of L955195 was much higher than that of Changwu9945-10 at each spike development stage. In L955195, during the spike development, the spike waxes were dominated by diketones (approximately 52 71\%). Differently, in Changwu9945-10, the spike waxes were dominated by alkanes (38 51\%) (Fig. 5). Similarly, the content of total wax on the leaves of L955195 was also much higher than that of Changwu994510 at each leaf development stage (Fig. 6). In Changwu9945-10, alcohols were the major compound during the leaf development while in L955195 alcohols were the major compound during the 50 to $100 \mathrm{~d}$, and then diketones were the major compound during the 200 to $230 \mathrm{~d}$. These results demonstrated that the composition of cuticular wax differed at different development stages and the trend of the changes were also different between glossy variety and glaucous variety.

Another interesting finding of this study was that the glaucous variety showed the presence of $\mathrm{OH}-\beta$-diketones presented in cuticular waxes. Consequently, based on the above discussion, it is inferred that $\beta$-diketones and $\mathrm{OH}-\beta$ diketones maybe synthesized by different genes, meanwhile, Changwu9945-10 and L955195 are useful wheat varieties for studies on the biosynthesis of diketones in wheat. Although $\beta$-diketones and $\mathrm{OH}-\beta$-diketones have been found in many plants (Adamski et al. 2013; Zhang et al. 2013; Wang et al. 2015a; Wang et al. 2017), there is still a lack of a broader understanding of its biological activity and its underlying mechanisms. Further research should be conducted to clarify the relevant genes and functions in different varieties, more and more additional physiological experiments are needed for future research.

\section{Conclusion}

Wax crystals were mainly comprised of tubules on the spike (glume) and platelets on the leaf surfaces. The OH- $\beta$ diketones were the major compounds that contributed to the glaucous phenotype in wheat, and the appearance of tubule wax crystals was based on high amounts of $\beta$-diketones. The constituents of the wax compound classes differed dramatically during the plant growth period. The homologs of compound classes of cuticular wax were also changed during the plant growth period. At the same time, the patterns of wax formation were different among varieties and organs of wheat.

\section{Acknowledgements}

Sincerely thank Zhonghua Wang (State Key Laboratory of Crop Stress Biology for Arid Areas, College of Agronomy, Northwest A\&F University, Yangling, Shaanxi, China) for his help for this research. This research was supported by Henan Wheat Industry Technical System Construction Special Fund Support Project (Z2010-01-04), Major Scientific Research Projects Focus on Special of Henan Academy of Sciences (200104003) the Key R\&D and Promotion Program of Henan Province (202102110028) and Basic Scientific Research Project of Henan Academy of Sciences (200604010). 


\section{Author Contributions}

J Wang and J Zhang planned the experiments, J Wang, J Fan and B Yang interpreted the results, J Wang, F Zhang and $\mathrm{Z}$ Cheng made the write up and $\mathrm{X}$ Chen statistically analyzed the data and made illustrations.

\section{Conflict of Interest}

The authors of this article have no conflict of interest of any kind

\section{Data Availability Declaration}

The authors declare that data reported in this article are available with the corresponding author and will be produced on demand

\section{References}

Adamski NM, MS Bush, J Simmonds, AS Turner, SG Mugford, A Jones (2013). The Inhibitor of wax 1 locus (Iw1) prevents formation of $\beta$ and $\mathrm{OH}-\beta$-diketones in wheat cuticular waxes and maps to a sub cM interval on chromosome arm 2BS. Plant J 74:989-1002

BelgeB, M Llovera, E Comabella, F Gatius, P Guillen, J Graell, I Lara (2014). Characterization of cuticle composition after cold storage of "Celeste" and "Somerset" sweet cherry fruit. J Agric Food Chem 62:8722-8729

Bianchi G, E Lupotto, B Borghi, M Corbellini (1980). Cuticular wax of wheat: The effects of chromosomal deficiencies on the biosynthesis of wax components. Planta 148:328-331

Bianchi G, ML Figini (1986). Epicuticular waxes of glaucous and nonglaucous durum wheat lines. J Agric Food Chem 34:429-433

Chai G, C Li, F Xu, Y Li, X Shi, Y Wang, ZH Wang (2018). Three endoplasmic reticulum-associated fatty acyl-coenzyme a reductases were involved in the production of primary alcohols in hexaploid wheat (Triticum aestivum L.). BMC Plant Biol 18; Article 41

Domínguez E, JA Heredia-Guerrero, A Heredia (2011). The biophysical design of plant cuticles: An overview. New Phytol 189:938-949

Eigenbrode SD, KE Espelie (1995). Effects of plant epicuticular lipids on insect herbivores. Annu Rev Entomol 40:171-194

Javelle M, V Vernoud, PM Rogowsky, GC Ingram (2011). Epidermis: The formation and functions of a fundamental plant tissue. New Phytol 189:17-39

Jeffree CE (2006). The fine structure of the plant cuticle. In: Annual Plant Reviews 23: Biology of the Plant Cuticle, pp:11-125. Riederer M, C Müller (eds.). Blackwell, Oxford, UK

Jenks MA, EN Ashworth (1999). Plant epiculticular waxes: Function, production and genetics. Hortic Rev 23:1-68
Jetter R, L Kunst, AL Samuels (2006). Biology of the Plant Cuticle. In: Composition of Plant Cuticular Waxes, pp:145-181. Riederer M, C Müller (eds.). John Wiley \& Sons, Blackwell, Oxford, UK

Koch K, W Barthlott, S Koch, A Hommes, K Wandelt, W Mamdouh (2006). Structural analysis of wheat wax (Triticum aestivum, c.v. 'Naturastar' L.): From the molecular level to three dimensional crystals. Planta 223:258-270

Koch K, HJ Ensikat (2008). The hydrophobic coatings of plant surfaces: Epicuticular wax crystals and their morphologies, crystallinity and molecular self-assembly. Micron 39:759-772

Li T, Y Sun, T Liu, H Wu, ZH Wang (2019). TaCER1-1A is involved in cuticular wax alkane biosynthesis in hexaploid wheat and responds to plant abiotic stresses. Plant Cell Environ 42:3077-3091

Li Y, F Beisson, OM Pollard (2007). Monoacylglycerols are components of root waxes and can be produced in the aerial cuticle by ectopic expression of a suberin-associated acyltransferase. Plant Physiol 144:1267-1277

Racovita RC, S Hen-Avivi, JP Fernandez-Moreno, A Granell, A Aharoni, R Jetter (2007). Composition of cuticular waxes coating flag leaf blades and peduncles of Triticum aestivum $c v$. Bethlehem. Phytochemistry 130:182-192

Rapley LP, GR Allen, BM Potts (2004). Susceptibility of Eucalyptus globulus to Mnesampela privata defoliation in relation to a specific foliar wax compound. Chemoecology 14:157-163

Samuels L, L Kunst, R Jetter (2008). Sealing plant surfaces: Cuticular wax formation by epidermal cells. Annu Rev Plant Biol 59:683-707

Schulz S, C Arsene, M Tauber, JN Mcneil (2000). Composition of lipids from sunflower pollen (Helianthus annuus). Phytochemistry 54:325-336

Solovchenko A, M Merzlyak (2003). Optical properties and contribution of cuticle to UV protection in plants: Experiments with apple fruit. Photochem. Photobiol Sci 2:861-866

Wang Y, JH Wang, GQ Chai, CL Li, YG Hu, XH Chen, ZH Wang (2015a). Developmental changes in composition and morphology of cuticular waxes on leaves and spikes of glossy and glaucous wheat (Triticum aestivum L.). PLoS One 10; Article e 0141239

Wang Y, ML Wang, YL Sun, D Hegebarth, TT Li, R Jetter, ZH Wang (2015b). Molecular characterization of TaFAR1 involved in primary alcohol biosynthesis of cuticular wax in hexaploid wheat. Plant Cell Physiol 56:1944-1961

Wang ML, Wu HQ, Xu J, Li CL, Wang Y, ZH Wang (2017). Five fatty acyl-coenzyme reductases are involved in the biosynthesis of primary alcohols in Aegilops tauschii leaves. Front Plant Sci 8; Article 1012

Wang Y, S Jin, Y Xu, S Li, S Zhang, Z Yuan, J Li, Y Ni (2020). Overexpression of BnKCS1-1, BnKCS1-2, and BnCER1-2 promotes cuticular wax production and increases drought tolerance in Brassica napus. Crop J 8:26-37

Zhang ZZ, W Wang, WL Li (2013). Genetic interactions underlying the biosynthesis and inhibition of $\beta$-diketones in wheat and their impact on glaucousness and cuticle permeability. PLoS One 8; Article e54129 